Article

\title{
Solution of Differential Equations with Polynomial Coefficients with the Aid of an Analytic Continuation of Laplace Transform
}

\author{
Tohru Morita ${ }^{1, *}$ and Ken-ichi Sato ${ }^{2}$ \\ 1 Graduate School of Information Sciences, Tohoku University, Sendai 980-8577, Japan \\ 2 College of Engineering, Nihon University, Koriyama 963-8642, Japan; kensatokurume@ybb.ne.jp \\ * Correspondence: senmm@jcom.home.ne.jp; Tel.: +81-22-278-6186 \\ Academic Editor: Hari M. Srivastava \\ Received: 22 December 2015; Accepted: 11 March 2016; Published: 17 March 2016
}

\begin{abstract}
In a series of papers, we discussed the solution of Laplace's differential equation (DE) by using fractional calculus, operational calculus in the framework of distribution theory, and Laplace transform. The solutions of Kummer's DE, which are expressed by the confluent hypergeometric functions, are obtained with the aid of the analytic continuation (AC) of Riemann-Liouville fractional derivative (fD) and the distribution theory in the space $\mathcal{D}_{R}^{\prime}$ or the AC of Laplace transform. We now obtain the solutions of the hypergeometric $\mathrm{DE}$, which are expressed by the hypergeometric functions, with the aid of the AC of Riemann-Liouville $\mathrm{fD}$, and the distribution theory in the space $\mathcal{D}_{r, R}^{\prime}$, which is introduced in this paper, or by the term-by-term inverse Laplace transform of AC of Laplace transform of the solution expressed by a series.
\end{abstract}

Keywords: Kummer's differential equation; hypergeometric differential equation; distribution theory; operational calculus; fractional calculus; Laplace transform

\section{Introduction}

Stimulated by Yosida's work [1,2], in which the solution of Laplace's differential equation (DE) is obtained with the aid of the operational calculus of Mikusiński [3], we are concerned in [4-6], with the DE or fractional DE of the form:

$$
\sum_{l=0}^{m}\left(a_{l} t+b_{l}\right) \cdot{ }_{0} D_{R}^{l \sigma} u(t)=f(t), \quad t>0,
$$

where $\sigma=\frac{1}{2}$ or $\sigma=1, m=2$, and $a_{l} \in \mathbb{C}$ and $b_{l} \in \mathbb{C}$ are constants.

In solving the $\mathrm{DE}$, we assume that the solution $u(t)$ and the inhomogeneous part $f(t)$ for $t>0$ are expressed as a linear combination of

$$
g_{v}(t):=\frac{1}{\Gamma(v)} t^{v-1}
$$

for $v \in \mathbb{C} \backslash \mathbb{Z}_{<1}$, where $\Gamma(v)$ is the gamma function.

In $[5,6],{ }_{0} D_{R}^{\beta} u(t)$ is the analytic continuation (AC) of Riemann-Liouville fractional derivative (fD), which was introduced in $[7,8]$ and is reviewed in [9]. It is defined for $u(t)$ and $f(t)$ satisfying the following conditions.

Condition A. $u(t) H(t)$ and $f(t) H(t)$ are expressed as a linear combination of $g_{v}(t) H(t)$ for $v \in S$, where $S$ is an enumerable set of $v \in \mathbb{C} \backslash \mathbb{Z}_{<1}$ satisfying $\operatorname{Re} v>-M$ for some $M \in \mathbb{Z}_{>-1}$. 
We now adopt Condition A. We then express $u(t)$ as follows:

$$
u(t)=\sum_{v \in S} u_{v-1} g_{v}(t)=\sum_{v \in S} u_{v-1} \frac{1}{\Gamma(v)} t^{v-1},
$$

where $u_{v-1} \in \mathbb{C}$ are constants.

For $v \in \mathbb{C} \backslash \mathbb{Z}_{<1,0} D_{R}^{\beta} g_{v}(t)$ is defined such that

$$
{ }_{0} D_{R}^{\beta} g_{v}(t)= \begin{cases}g_{v-\beta}(t), & v-\beta \in \mathbb{C} \backslash \mathbb{Z}_{<1}, \\ 0, & v-\beta \in \mathbb{Z}_{<1} .\end{cases}
$$

When $\beta=n \in \mathbb{Z}_{>-1,0} D_{R}^{n} g_{v}(t)=\frac{d^{n}}{d t^{n}} g_{v}(t)$. Throughout the present paper, the equations involving $\beta$ are valid for $\beta \in \mathbb{C}$, but in the applications given in Sections $4-7$, we use them only for $\beta=n \in \mathbb{Z}_{>-1}$, when ${ }_{0} D_{R}^{n} g_{v}(t)=\frac{d^{n}}{d t^{n}} g_{v}(t)$.

We use $\mathbb{R}, \mathbb{C}$ and $\mathbb{Z}$ to denote the sets of all real numbers, of all complex numbers and of all integers, respectively. We also use $\mathbb{R}_{>r}:=\{x \in \mathbb{R} \mid x>r\}$ for $r \in \mathbb{R},{ }_{+} \mathbb{C}:=\{z \in \mathbb{C} \mid \operatorname{Re} z>0\}$, $\mathbb{Z}_{>a}:=\{n \in \mathbb{Z} \mid n>a\}, \mathbb{Z}_{<b}:=\{n \in \mathbb{Z} \mid n<b\}$ and $\mathbb{Z}_{[a, b]}:=\{n \in \mathbb{Z} \mid a \leq n \leq b\}$ for $a, b \in \mathbb{Z}$ satisfying $a<b$. We use Heaviside's step function $H(t)$, which is defined such that (i) $H(t)=1$ for $t>0$ and $=0$ for $t \leq 0$, and (ii) when $f(t)$ is defined on $\mathbb{R}_{>r}, f(t) H(t-r)$ is equal to $f(t)$ when $t>r$ and to 0 when $t \leq r$.

In [4-6], we take up a modified Kummer's DE as an example, which is

$$
t \cdot \frac{d^{2}}{d t^{2}} u(t)+(c-b t) \cdot \frac{d}{d t} u(t)-a b \cdot u(t)=0, \quad t>0
$$

where $a \in \mathbb{C}, b \in \mathbb{C}$ and $c \in \mathbb{C}$ are constants. Kummer's DE is this DE with $b=1[10,11]$. If $c \notin \mathbb{Z}$, the basic solutions of Equation (5) are given by

$$
\begin{aligned}
& u_{1}(t):={ }_{1} F_{1}(a ; c ; b t), \\
& u_{2}(t):=t^{1-c} \cdot{ }_{1} F_{1}(a-c+1 ; 2-c ; b t) .
\end{aligned}
$$

Here ${ }_{1} F_{1}(a ; c ; z)=\sum_{k=0}^{\infty} \frac{(a)_{k}}{k !(c)_{k}} z^{k}$ is the confluent hypergeometric series, where $z \in \mathbb{C}$, $(a)_{n}=\prod_{k=0}^{n-1}(a+k)$ for $a \in \mathbb{C}$ and $n \in \mathbb{Z}_{>0}$, and $(a)_{0}=1$. These solutions are expressed as linear combinations of $g_{v}(t)$.

Remark 1. In [4-6], $a, b$ and $c$ in Equation (5) are expressed as $\gamma_{1}+1,-\alpha$ and $\gamma_{1}+\gamma_{2}+2$, respectively.

In $[4,5]$, we consider the theory of distributions in the space $\mathcal{D}_{R}^{\prime}$, which is presented in $[12,13]$ and is explained briefly in Section 3.3. The solution of Equation (1) with the aid of distributions in $\mathcal{D}_{R}^{\prime}$ is presented in [5], assuming that the solution satisfies Condition A. Both of the solutions given by Equations (6) and (7), of Equation (5), satisfy Condition A, and we can obtain both of them, by solving Equation (5) by this method.

In [4], we adopt the following condition.

Condition B. $u(t) H(t)$ and $f(t) H(t)$ are expressed as a linear combination of $g_{v}(t) H(t)$ for $v \in S_{1}$, where $S_{1}$ is a set of $v \in{ }_{+} \mathbb{C}$.

When Condition B is satisfied, ${ }_{0} D_{R}^{\beta} u(t)$ of a function $u(t)$ denotes the Riemann-Liouville fD which is defined when $u(t) H(t)$ is locally integrable on $\mathbb{R}$, and hence ${ }_{0} D_{R}^{\beta} g_{v}(t)$ is defined only for $v \in+\mathbb{C}$, satisfying Equation (4). In this case, the DE given by Equation (1) in terms of the distribution theory in space $\mathcal{D}_{R}^{\prime}$ is presented in [4]. The solutions of fractional DE with constant coefficients are 
presented in [12,13]. In [4], the solution given by Equation (6) of Equation (5), satisfies Condition B, and hence we can obtain it by solving Equation (5) by this method. However, the solution given by Equation (7) satisfies Condition B only when $1-c>-1$, and hence we can obtain it only when $1-c>-1$, by this method.

Condition C. There exists $\lambda \in \mathbb{R}_{>0}$ such that $u(t) e^{-\lambda t} \rightarrow 0$ as $t \rightarrow \infty$.

In [6], it was mentioned that, when Conditions B and C are satisfied, the Laplace transform of $u(t)$ exists and the DE is solved with the aid of Laplace transform, and that the solutions of Equation (5), satisfying Condition B, satisfy Condition C and hence are obtained by using the Laplace transform.

In [6], the AC of Laplace (AC-Laplace) transform is introduced as in Section 1.1 given below, and it is shown that, when Conditions A and C are satisfied, the AC-Laplace transform of $u(t)$, which is denoted by $\hat{u}(s)=\mathcal{L}_{H}[u(t)]=\mathcal{L}_{H}[u(t)](s)$, exists and the DE given by Equation (1) is solved with the aid of the AC-Laplace transform. In fact, the AC-Laplace transform of $g_{v}(t)$ and of $u(t)$ given by Equation (3) are expressed as

$$
\begin{gathered}
\hat{g}_{v}(s)=\mathcal{L}_{H}\left[g_{v}(t)\right]=s^{-v}, \quad v \in \mathbb{C} \backslash \mathbb{Z}_{<1}, \\
\hat{u}(s)=\mathcal{L}_{H}[u(t)]=\sum_{v \in S} u_{v-1} \hat{g}_{v}(s) .
\end{gathered}
$$

We review the solution in terms of the AC-Laplace transform in Section 2, and the solution with the aid of the distribution theory in Section 3. In Section 4 we confirm the following lemma.

Lemma 1. Both of the solutions given by Equations (6) and (7), of Equation (5), satisfy Conditions A and $C$, and hence we can obtain both of them, by solving Equation (5) by using distribution theory in the space $\mathcal{D}_{R}^{\prime}$ and also by using the AC-Laplace transform.

In Section 5, we consider the hypergeometric $\mathrm{DE}$, which is given by

$$
t(1-t) \cdot \frac{d^{2}}{d t^{2}} u(t)+(c-(a+b+1) t) \cdot \frac{d}{d t} u(t)-a b \cdot u(t)=0, \quad t>0,
$$

where $a \in \mathbb{C}, b \in \mathbb{C}$ and $c \in \mathbb{C}$ are constants.

If $c \notin \mathbb{Z}$, the basic solutions of Equation (10) in $[10,11]$ are given by

$$
\begin{aligned}
& u_{1}(t):={ }_{2} F_{1}(a, b ; c ; t), \\
& u_{2}(t):=t^{1-c} \cdot{ }_{2} F_{1}(1+a-c, 1+b-c ; 2-c ; t),
\end{aligned}
$$

where ${ }_{2} F_{1}(a, b ; c ; z)=\sum_{n=0}^{\infty} \frac{(a)_{n}(b)_{n}}{n !(c)_{n}} z^{n}$ of $z \in \mathbb{C}$ is the hypergeometric series.

Remark 2. These solutions of Equation (10) converge only at $t$ satisfying $|t|<1$, and do not satisfy Condition A, and they are not obtained by the methods stated above.

We introduce the theory of distributions in the space $\mathcal{D}_{r, R}^{\prime}$, in Section 3.4. We now use the step function $H_{r}(t)$, which is defined for $r \in \mathbb{R}_{>0}$ such that (i) $H_{r}(t)=1$ for $0<t<r$ and $=0$ for $t \leq 0$ or $t \geq r$, and (ii) when $f(t)$ is defined on $\mathbb{R}_{>0} \cap \mathbb{R}_{<r}, f(t) H_{r}(t)$ is equal to $f(t)$ for $0<t<r$ and to 0 for $t \leq 0$ or $t \geq r$.

Condition D. Condition A with $H(t)$ replaced by $H_{r}(t)$ is valid. 
In Section 5, we show that when $u(t)$ satisfies Condition D, we can solve Equation (10) with the aid of distributions in $\mathcal{D}_{r, R}^{\prime}$.

Definition 1. Let $u(t)$ be given by Equation (3) and satisfy Condition D. Then, we define its AC of Riemann-Liouville $\mathrm{fD}$ of order $\beta \in \mathbb{C}$, by

$$
{ }_{0} D_{R}^{\beta} u(t)=\sum_{v \in S, v-\beta \notin \mathbb{Z}_{<1}} u_{v-1} g_{v-\beta}(t),
$$

which satisfies Condition D.

Definition 2. Let $u(t)$ be given by Equation (3) and satisfy Condition D. Then, we define $\hat{u}(s)=$ $\mathcal{L}_{S}[u(t)]=\mathcal{L}_{S}[u(t)](s)$ by

$$
\hat{u}(s)=\mathcal{L}_{S}[u(t)]=\sum_{v \in S} u_{v-1} \hat{g}_{v}(s)
$$

We call this the AC-Laplace transform series of $u(t)$.

For the solutions of Equation (10), the existence of the AC-Laplace transform is not guaranteed, but we can define $\hat{u}(s)$ by Equation (14). We can then set up a DE satisfied by the thus-defined $\hat{u}(s)$. In Section 5, we write the DE for the $\hat{u}(s)$, and its solution in the form of Equation (14) is obtained. We find that the obtained series converges for no value of $s$, and yet we obtain the solution $u(t)$ by the term-by-term inverse Laplace transform of the series $\hat{u}(s)$.

The solutions given by Equations (11) and (12), of Equation (10), satisfy Condition D for $r=1$, and we show that they are obtained by using the distribution theory in the space $\mathcal{D}_{r, R}^{\prime}$ and also by using the AC of Laplace transform series, in Section 5.

In Section 6, we show that the Bessel functions $J_{ \pm v}(t)$ are the solusions of Bessel's DE with the aid of the AC-Laplace transform. In Section 7, some discussions are given on Hermite's DE. Concluding remarks are given in Section 8.

\subsection{Definition of the AC-Laplace Transform}

The AC-Laplace transform $\hat{f}(s)=\mathcal{L}_{H}[f(t)]$ of a function $f(t)$ is defined in [6] as follows.

Condition E. $f_{\gamma}(z)$ is expressed as $f_{\gamma}(z)=z^{\gamma-1} f_{1}(z)$ on a neighborhood of $\mathbb{R}_{>0}$, for $0 \leq \arg z<2 \pi$, where $\gamma \in \mathbb{C} \backslash \mathbb{Z}_{<1}$, and $f_{1}(z)$ is analytic on the neighborhood of $\mathbb{R}_{>0}$.

Definition 3. Let $f_{\gamma}(z)$ and $u(t)=f_{\gamma}(t)$ satisfy Conditions E and C, respectively. Then, we define the AC-Laplace transform $\hat{f}_{\gamma}(s)$ for $\gamma \in \mathbb{C} \backslash \mathbb{Z}_{<1}$, by $\hat{f}_{\gamma}(s)=\mathcal{L}_{H}\left[f_{\gamma}(t)\right]$, where

$$
\mathcal{L}_{H}\left[f_{\gamma}(t)\right]=e^{-i \pi \gamma} \frac{1}{2 i \sin \pi \gamma} \int_{C_{H}} f_{\gamma}(\zeta) e^{-s \zeta} d \zeta, \quad \gamma \in \mathbb{C} \backslash \mathbb{Z}
$$

When $\gamma=n \in \mathbb{Z}_{>0}$, we put $\mathcal{L}_{H}\left[f_{n}(t)\right]=\lim _{\gamma_{i} \rightarrow n} \mathcal{L}_{H}\left[t \gamma_{i}-1 \cdot f_{1}(t)\right]$, where $\gamma_{i} \in \mathbb{C} \backslash \mathbb{Z}$. Here, $C_{H}$ is the contour which appears in Hankel's formula giving the $\mathrm{AC}$ of the gamma function $\Gamma(z)$, so that $C_{H}$ is the contour which starts from $\infty+i \epsilon$, goes to $\delta+i \epsilon$, encircles the origin counterclockwise, goes to $\delta-i \epsilon$, and then to $\infty-i \epsilon$, where $\delta \in \mathbb{R}_{>0}$ and $\epsilon \in \mathbb{R}_{>0}$ satisfy $\epsilon \leqq 1$ and $\delta<1$, see [14] (Section 12.22).

Remark 3. $\hat{f}_{\gamma}(s)$ defined by Definition 3 is an analytic continuation of the Laplace transform defined by $\hat{f}_{\gamma}(s)=\int_{0}^{\infty} f_{\gamma}(t) e^{-s t} d t$ for $\operatorname{Re} \gamma>0$, as a function of $\gamma$. 


\subsection{Remarks on Recent Developments}

Here, we call attention to recent developments on the solutions of differential equations related with fractional calculus and perturbation method, which are based on He's variational iteration method (VIM) [15]. By using the VIM, He gave the fD and fI which involve the terms determined by the initial or boundary condition. Liu et al. [16] discussed the solution of heat conduction in a fractal medium with the aid of He's fD. Kumar et al. discussed the solution of partial differential equations involving time-fD by using Laplace transform and perturbation method based on the VIM; see [17,18] and references in them. In $[19,20]$, discussions are given on the fractional complex transform, which reduces an equation involving $\mathrm{fD}$ to an equation involving only integer-order derivatives.

\section{AC-Laplace Transform}

In the present section and Section 2.1, we assume that $u(t)$ satisfies Conditions $\mathrm{C}$ and $\mathrm{A}$, and we put $\hat{u}(s)=\mathcal{L}_{H}[u(t)]$.

Lemma 2. Let $v \in \mathbb{C} \backslash \mathbb{Z}_{<1}$ and $n \in \mathbb{Z}_{>-1}$. Then

$$
\begin{aligned}
t^{n} \cdot g_{v}(t) & =(v)_{n} \cdot g_{v+n}(t), \\
(-1)^{n} \frac{d^{n}}{d s^{n}} \hat{g}_{v}(s) & =(v)_{n} \cdot \hat{g}_{v+n}(s),
\end{aligned}
$$

where $(v)_{n}$ is Pochhammer's symbol, so that $(v)_{n}=\frac{\Gamma(v+n)}{\Gamma(v)}$.

Proof We confirm Equation (16) by using Equation (2) on both sides of Equation (16). By Equation (8), $\mathcal{L}_{H}\left[g_{v}(t)\right]=\hat{g}_{v}(s)=s^{-v}$. By taking derivatives of this equation repeatedly, we obtain Equation (17).

Lemma 3. Let $v \in \mathbb{C} \backslash \mathbb{Z}_{<1}, n \in \mathbb{Z}_{>-1}$ and $\beta \in \mathbb{C}$. Then

$$
\mathcal{L}_{H}\left[t^{n} \cdot g_{v}(t)\right]=(-1)^{n} \frac{d^{n}}{d s^{n}} \hat{g}_{v}(s)
$$

Proof Equation (18) is obtained by comparing Equations (16) and (17) with Equation (8).

Applying Lemma 3 to Equation (4), with the aid of Equation (8), we obtain

$$
\mathcal{L}_{H}\left[t^{n} \cdot{ }_{0} D_{R}^{\beta} g_{v}(t)\right]= \begin{cases}(-1)^{n} \frac{d^{n}}{d s^{n}} \hat{g}_{v-\beta}(s)=(-1)^{n} \frac{d^{n}}{d s^{n}}\left[s^{\beta} \hat{g}_{v}(s)\right], & v-\beta \in \mathbb{C} \backslash \mathbb{Z}_{<1}, \\ 0, & v-\beta \in \mathbb{Z}_{<1} .\end{cases}
$$

Lemma 4. Let $v \in \mathbb{C} \backslash \mathbb{Z}_{<1}, n \in \mathbb{Z}_{>-1}$ and $\beta \in \mathbb{C}$. Then

$$
\mathcal{L}_{H}\left[t^{n} \cdot{ }_{0} D_{R}^{\beta} g_{v}(t)\right]=(-1)^{n} \frac{d^{n}}{d s^{n}}\left[s^{\beta} \hat{g}_{v}(s)\right]-(-1)^{n} \frac{d^{n}}{d s^{n}}\left\langle s^{\beta} \hat{g}_{v}(s)\right\rangle_{z},
$$

where

$$
\left\langle s^{\beta} \hat{g}_{v}(s)\right\rangle_{z}= \begin{cases}0, & v-\beta \in \mathbb{C} \backslash \mathbb{Z}_{<1}, \\ s^{\beta} \hat{g}_{v}(s)=\hat{g}_{-k}(s)=s^{k}, & -k=v-\beta \in \mathbb{Z}_{<1} .\end{cases}
$$

Proof Here, $\left\langle s^{\beta} \hat{g}_{v}(s)\right\rangle_{z}$ is so defined that Equation (20) with Equation (21) represents Equation (19). 
Lemma 5. Let $u(t)$ be expressed by Equation (3), $n \in \mathbb{Z}_{>-1}$ and $\beta \in \mathbb{C}$. Then

$$
\begin{aligned}
\mathcal{L}_{H}\left[t^{n} \cdot u(t)\right] & =(-1)^{n} \frac{d^{n}}{d s^{n}} \hat{u}(s), \\
\mathcal{L}_{H}\left[t^{n} \cdot{ }_{0} D_{R}^{\beta} u(t)\right] & =(-1)^{n} \frac{d^{n}}{d s^{n}}\left[s^{\beta} \hat{u}(s)\right]-\left\langle(-1)^{n} \frac{d^{n}}{d s^{n}}\left[s^{\beta} \hat{u}(s)\right]\right\rangle_{z},
\end{aligned}
$$

where

$$
\left\langle(-1)^{n} \frac{d^{n}}{d s^{n}}\left[s^{\beta} \hat{u}(s)\right]\right\rangle_{z}=(-1)^{n} \frac{d^{n}}{d s^{n}}\left\langle s^{\beta} \hat{u}(s)\right\rangle_{z}, \quad\left\langle s^{\beta} \hat{u}(s)\right\rangle_{z}=\sum_{\substack{v \in S \\-k=\nu-\beta \in \mathbb{Z}_{<1}}} u_{v-1} s^{k} .
$$

Proof We confirm these with the aid of Equation (9) and Lemmas 3 and 4.

Remark 4. Let $\beta=n \in \mathbb{Z}_{>-1}$. Then, Equation (24) gives $\langle\hat{u}(s)\rangle_{z}=0$, and

$$
\left\langle s^{n} \hat{u}(s)\right\rangle_{z}=\sum_{\substack{k=0 \\ n-k \in S}}^{n-1} u_{n-k-1} s^{k}, \quad n \in \mathbb{Z}_{>0}
$$

so that

$$
\langle\hat{u}(s)\rangle_{z}=0, \quad\langle s \cdot \hat{u}(s)\rangle_{z}=u_{0}, \quad\left\langle s^{2} \cdot \hat{u}(s)\right\rangle_{z}=u_{1}+u_{0} s, \quad\left\langle s^{3} \cdot \hat{u}(s)\right\rangle_{z}=u_{2}+u_{1} s+u_{0} s^{2}, \cdots .
$$

Theorem 1. Let $u(t)$ satisfy Conditions $C$ and $A$, and be expressed by Equation (3). Then, the AC-Laplace transform $\hat{u}(s)$ is expressed as Equation (9) and there exists $M \in \mathbb{R}_{>0}$ such that the series given by Equation (9) converges for $|s|>M$.

\subsection{Recipe of Solving Differential Equation with Polynomial Coefficients}

We now give a recipe of solving the DE with polynomial coefficients, which is given by

$$
\sum_{l=0}^{2}\left(a_{l} t^{2}+b_{l} t+c_{l}\right) \cdot \frac{d^{l}}{d t^{l}} u(t)=f(t), \quad t>0,
$$

where $a_{l} \in \mathbb{C}, b_{l} \in \mathbb{C}$ and $c_{l} \in \mathbb{C}$ for $l \in \mathbb{Z}_{[0,2]}$ are constants. We then introduce the function $p(t, s)$ by

$$
p(t, s)=\sum_{l=0}^{2}\left(a_{l} t^{2}+b_{l} t+c_{l}\right) \cdot s^{l}
$$

and express Equation (27) as

$$
p\left(t, \frac{d}{d t}\right) u(t)=f(t), \quad t>0 .
$$

In Sections 4 and 5, we discuss modified Kummer's DE given by Equation (5) and the hypergeometric DE given by Equation (10).

We obtain the following theorems, with the aid of Lemma 5.

Theorem 2. Let $u(t)$ in the form of Equation (3) be the solution of Equation (29). Then $\hat{u}(s)$ given by Equation (9) is a solution of the DE:

$$
p\left(-\frac{d}{d s}, s\right) \hat{u}(s)=\hat{f}(s)+\left\langle p\left(-\frac{d}{d s}, s\right) \hat{u}(s)\right\rangle_{z} .
$$


Theorem 3. Let $\hat{u}(s)$ in the form of Equation (9) be a solution of Equation (30). Then, the corresponding $u(t)$ given by Equation (3) is a solution of Equation (29).

Corollary 4. Let $\hat{u}(s)$ be a solution of

$$
p\left(-\frac{d}{d s}, s\right) \hat{u}(s)=\hat{f}(s)
$$

If the obtained $\hat{u}(s)$ satisfies

$$
\left\langle p\left(-\frac{d}{d s}, s\right) \hat{u}(s)\right\rangle_{z}=0
$$

then $u(t)$ given by Equation (3) is a solution of Equation (29), that is of Equation (27).

\subsection{Term-by-Term Operators for $u(t)$ and $\hat{u}(s)$}

The AC of Riemann-Liouville fD: ${ }_{0} D_{R}^{\beta} u(t)$ and the AC-Laplace transform series: $\mathcal{L}_{S}[u(t)]$, of $u(t)$ in the form of Equation (3), are defined by Equation (13) and Equation (14). We now define the operators which appear in Lemma 5.

Definition 4. Let $n \in \mathbb{Z}_{>0}, \beta \in \mathbb{C}$ and $\gamma \in \mathbb{C}$, and let $u(t)$ and $\hat{u}(s)$ be expressed as Equation (3) and Equation (14), respectively. Then, we adopt

$$
\begin{aligned}
t^{n} \cdot u(t) & =\sum_{v \in S}\left(t^{n} \cdot u_{v-1} g_{v}(t)\right), \\
s^{\beta} \hat{u}(s) & =\sum_{v \in S}\left(s^{\beta} \cdot u_{v-1} \hat{g}_{v}(s)\right), \quad s^{\gamma}\left(s^{\beta} \hat{u}(s)\right)=s^{\gamma+\beta} \hat{u}(s), \\
(-1)^{n} \frac{d^{n}}{d s^{n}}\left[s^{\beta} \hat{u}(s)\right] & =\sum_{v \in S}\left[(-1)^{n} \frac{d^{n}}{d s^{n}}\left(s^{\beta} u_{v-1} \hat{g}_{\nu}(s)\right)\right] .
\end{aligned}
$$

Proposition 5. Let the operators on the lhs of the equations in Definition 2, 3 and 4 be defined by the respective rhs. Then, Theorems 2 and 3 and Corollary 4 are valid.

\section{Operational Calculus in the Spaces $\mathcal{D}_{R}^{\prime}$ and $\mathcal{D}_{r, R}^{\prime}$}

We now consider the theory of distributions in the spaces $\mathcal{D}_{R}^{\prime}$ and $\mathcal{D}_{r, R}^{\prime}$, which are explained briefly in Sections 3.3 and 3.4, respectively.

\subsection{Operational Calculus in the Spaces $\mathcal{D}_{R}^{\prime}$}

In the theory of distributions in $\mathcal{D}_{R}^{\prime}$, a regular distribution is such a distribution that can be regarded as a function which is locally integrable on $\mathbb{R}$. In the present paper, when the product $u(t) H(t) \in \mathcal{L}_{\text {loc }}(\mathbb{R})$, we consider the regular distribution $\tilde{u}(t)$ which is regarded to be equal to $u(t) H(t)$. We then denote this correspondence between a distribution and a function by $\tilde{u}(t) \bullet-\circ u(t) H(t)$. Here, $u(t) H(t) \in \mathcal{L}_{l o c}(\mathbb{R})$ denotes that $u(t) H(t)$ is locally integrable on $\mathbb{R}$. From Equation (2), we have $g_{1}(t)=1$. We put $\tilde{H}(t)=\tilde{g}_{1}(t)$, and then we obtain $\tilde{H}(t) \bullet-\circ$ $g_{1}(t) H(t)=H(t)$.

Definition 5. In the space $\mathcal{D}_{R}^{\prime}$, the differential operator $D^{\lambda}$ of order $\lambda \in \mathbb{C}$ is defined as follows:

(i) If $\operatorname{Re} v>0$ and $u(t) H(t) \in \mathcal{L}_{\text {loc }}(\mathbb{R})$, then $D^{-v} \tilde{u}(t) \bullet-\circ \quad\left[{ }_{0} D_{R}^{-v} u(t)\right] H(t)$.

(ii) If $h(t) \in \mathcal{D}_{R}^{\prime}$, then $D^{\lambda} h(t) \in \mathcal{D}_{R}^{\prime}$.

(iii) The index law stated in the following lemma is valid. 
Lemma 6. Let $h(t) \in \mathcal{D}_{R}^{\prime}$. Then, the index law:

$$
D^{\lambda} D^{\mu} h(t)=D^{\lambda+\mu} h(t)
$$

is valid for every pair of $\lambda \in \mathbb{C}$ and $\mu \in \mathbb{C}$.

Dirac's delta function $\delta(t) \in \mathcal{D}_{R}^{\prime}$ is the distribution defined by $\delta(t)=D \tilde{H}(t)$.

We now introduce the correspondence even between a distribution, which is not a regular one, and a function, as follows.

Definition 6. Let $v \in \mathbb{C}, u(t)$ and $v(t)={ }_{0} D_{R}^{v} u(t)$ satisfy $u(t) H(t) \in \mathcal{L}_{\text {loc }}(\mathbb{R})$ and ${ }_{0} D_{R}^{-v} v(t)=u(t)$. Then, $\tilde{v}(t)=D^{v} \tilde{u}(t) \bullet{ }_{0} D_{R}^{v} u(t) H(t)=v(t) H(t)$.

We now define $\tilde{g}_{v}(t)$ for $v \in \mathbb{C}$ by

$$
\tilde{g}_{v}(t)=D^{-v+1} \tilde{H}(t)=D^{-v} \delta(t)=\hat{g}_{v}(D) \delta(t) .
$$

From Equations (2) and (4), we have $g_{1}(t)=1, g_{v}(t)={ }_{0} D_{R}^{-v+1} g_{1}(t)$ and $g_{1}(t)={ }_{0} D_{R}^{v-1} g_{v}(t)$ for $v \in \mathbb{C} \backslash \mathbb{Z}_{<1}$. Since $g_{1}(t) H(t)=H(t) \in \mathcal{L}_{\text {loc }}(\mathbb{R})$, by Definition 6, we have

$$
\tilde{g}_{v}(t)=\hat{g}_{v}(D) \delta(t) \bullet g_{v}(t) H(t), \quad v \in \mathbb{C} \backslash \mathbb{Z}_{<1} .
$$

Remark 5. Note here that when $\operatorname{Re} v \leq-1, \tilde{g}_{v}(t)$ is not a regular distribution.

Lemma 7. Let $v \in \mathbb{C} \backslash \mathbb{Z}_{<1}$ and $n \in \mathbb{Z}_{>-1}$. Then

$$
(-1)^{n} \frac{\partial^{n}}{\partial D^{n}} \tilde{g}_{v}(t)=(-1)^{n} \frac{\partial^{n}}{\partial D^{n}} \hat{g}_{v}(D) \delta(t) \bullet\left[\quad t^{n} \cdot g_{v}(t)\right] H(t),
$$

where the derivative with respect to $D$ is taken regarding $D$ as a variable.

Proof By comparing Equation (18) with Equation (38), we confirm this.

By Equations (37) and (36), we have

$$
D^{\beta} \tilde{g}_{v}(t)=D^{\beta-v} \delta(t)=\tilde{g}_{v-\beta}(t) .
$$

Comparing this with Equations (4) and (38), we have

Lemma 8. Let $v \in \mathbb{C} \backslash \mathbb{Z}_{<1}$ and $\beta \in \mathbb{C}$. Then

$$
D^{\beta} \tilde{g}_{v}(t)-\left\langle D^{\beta} \tilde{g}_{v}(t)\right\rangle_{z}=D^{\beta} \hat{g}_{v}(D) \delta(t)-\left\langle D^{\beta} \hat{g}_{v}(D)\right\rangle_{z} \delta(t) \bullet \multimap\left[{ }_{0} D_{R}^{\beta} g_{v}(t)\right] H(t),
$$

where

$$
\left\langle D^{\beta} \tilde{g}_{v}(t)\right\rangle_{z}=\left\langle D^{\beta} \hat{g}_{v}(D)\right\rangle_{z} \delta(t)= \begin{cases}0, & v-\beta \in \mathbb{C} \backslash \mathbb{Z}_{<1}, \\ D^{\beta} \tilde{g}_{v}(t)=\tilde{g}_{-k}(t)=D^{k} \delta(t), & -k=v-\beta \in \mathbb{Z}_{<1} .\end{cases}
$$

Definition 7. Let $u(t)$ be expressed by Equation (3). Then, we define the distribution $\tilde{u}(t)$ by

$$
\tilde{u}(t):=\hat{u}(D) \delta(t)=\sum_{v \in S} u_{v-1} \tilde{g}_{v}(t)=\sum_{v \in S} u_{v-1} \hat{g}_{v}(D) \delta(t),
$$

in accordance with Equation (37). 
Definition 8. We adopt Definition 4 with $\hat{u}(s), \hat{g}_{v}(s), s$ and $\frac{d}{d s}$ replaced by $\tilde{u}(t)=\hat{u}(D) \delta(t)$, $\tilde{g}_{v}(t)=\hat{g}_{v}(D) \delta(t), D$ and $\frac{\partial}{\partial D}$, respectively.

Lemma 9. Let $u(t)$ be expressed by Equation (3), $n \in \mathbb{Z}_{>-1}$ and $\beta \in \mathbb{C}$. Then

$$
\begin{aligned}
&(-1)^{n} \frac{\partial^{n}}{\partial D^{n}} \tilde{u}(t)=(-1)^{n} \frac{\partial^{n}}{\partial D^{n}} \hat{u}(D) \delta(t) \bullet\left[t^{n} \cdot u(t)\right] H(t), \\
&(-1)^{n} \frac{\partial^{n}}{\partial D^{n}} D^{\beta} \tilde{u}(t)-\left\langle(-1)^{n} \frac{\partial^{n}}{\partial D^{n}} D^{\beta} \tilde{u}(t)\right\rangle_{z}=(-1)^{n} \frac{\partial^{n}}{\partial D^{n}}\left[D^{\beta} \hat{u}(D)\right] \delta(t)-\left\langle(-1)^{n} \frac{\partial^{n}}{\partial D^{n}}\left[D^{\beta} \hat{u}(D)\right]\right\rangle_{z} \delta(t) \\
& \bullet \bullet\left[t^{n} \cdot{ }_{0} D_{R}^{\beta} u(t)\right] H(t),
\end{aligned}
$$

where $\left\langle D^{\beta} \hat{u}(D)\right\rangle_{z}$ and $\left\langle(-1)^{n} \frac{\partial^{n}}{\partial D^{n}}\left[D^{\beta} \hat{u}(D)\right]\right\rangle_{z}$ are defined by Equation (24) with $-\frac{d}{d s}$ and $s$ replaced by $-\frac{\partial}{\partial D}$ and $D$, respectively.

Proof The first equalities in Equations (44) and (45) are due to Definition 7. When $u(t)=g_{v}(t)$ for $v \in \mathbb{C} \backslash \mathbb{Z}_{<-1}$, Equation (44) is Equation (39). Equation (45) for $n=0$ in this case is Equation (41). Equation (45) for $n>0$ in this case is derived by applying Equation (44) to Equation (41). When $u(t)$ is expressed by Equation (3), we confirm Equations (44) and (45) by using the rhs of Equations (43), (14) and (3) in place of $\tilde{u}(t), \hat{u}(D)$ and $u(t)$, respectively, in Equations (44) and (45), and comparing the respective equations for the case of $u(t)=g_{v}(t)$ for $v \in \mathbb{C} \backslash \mathbb{Z}_{<1}$.

\subsubsection{Recipe of Solving Linear Differential Equation with Polynomial Coefficients}

By applying Lemma 9 to the DE given by Equation (29), we obtain the following lemma.

Lemma 10. The $\mathrm{DE}$ for $\tilde{u}(t)=\hat{u}(D) \delta(t)$ corresponding to Equation (29) is

$$
p\left(-\frac{\partial}{\partial D}, D\right) \hat{u}(D) \delta(t)=\tilde{f}(t)+\left\langle p\left(-\frac{\partial}{\partial D}, D\right) \hat{u}(D)\right\rangle_{z} \delta(t) .
$$

Theorem 6. When $\tilde{u}(t)=\hat{u}(D) \delta(t)$ is a solution of Equation (46), the corresponding $u(t)$ is a solution of Equation (29).

Proposition 7. Let $\hat{u}(s)$ be a solution of Equation (30), and $\tilde{u}(t)=\hat{u}(D) \delta(t)$ obtained from it be expressed in the form of Equation (43). Then $u(t)$ given by Equation (3) is a solution of Equation (29).

Corollary 8. Let $\hat{u}(s)$ be a solution of Equation (31) and $\tilde{u}(t)=\hat{u}(D) \delta(t)$, which is obtained from it, be expressed in the form of Equation (43). If the obtained $\tilde{u}(t)$ satisfies

$$
\left\langle p\left(-\frac{\partial}{\partial D}, D\right) \hat{u}(D)\right\rangle_{z} \delta(t)=0,
$$

then $u(t)$ given by Equation (3) is a solution of Equation (29).

\subsection{Operational Calculus in the Space $\mathcal{D}_{r, R}^{\prime}$}

For the function $H_{r}(t)$ defined below Equation (12) in the Introduction section, we define the regular distribution $\tilde{H}_{r}(t)$ in the space $\mathcal{D}_{r, R}^{\prime}$, so that $\tilde{H}_{r}(t) \bullet g_{1}(t) H_{r}(t)=H_{r}(t)$, and then define $\delta_{r}(t) \in \mathcal{D}_{r, R}^{\prime}$ by $\delta_{r}(t)=D \tilde{H}_{r}(t)=\delta(t)-\delta(t-r)$. We can use the formulas presented in Section 3.1 for the space $\mathcal{D}_{R}^{\prime}$, also in the space $\mathcal{D}_{r, R}^{\prime}$, if we replace $H(t), \tilde{H}(t)$ and $\delta(t)$, by $H_{r}(t), \tilde{H}_{r}(t)$ and $\delta_{r}(t)$, respectively.

In Section 4, Theorem 1 applies. In Section 5, the rhs of Equation (44) for $n=0$ is $u(t) H_{r}(t)=$ $\sum_{v \in S} u_{v-1} g_{v}(t) H_{r}(t)$, which converges at $|t|<1$. Although $\hat{u}(s)$ converges for no value of $s$, we 
regard that the rhs of Equation (44) converges at $|t|<1$, and the lhs of Equation (44) represents the corresponding regular distribution. The result obtained for $u(t)$ is then the one obtained by the term-by-term inverse Laplace transform of $\hat{u}(s)$, which is expressed as Equation (14).

\subsection{Distributions in the Space $\mathcal{D}_{R}^{\prime}$}

Distributions in the space $\mathcal{D}^{\prime}$ are first introduced [21-24]. The distributions are either regular ones or their derivatives. A regular distribution in $\mathcal{D}^{\prime}$ corresponds to a function which is locally integrable on $\mathbb{R}$. The space $\mathcal{D}$, that is dual to $\mathcal{D}^{\prime}$, is the space of testing functions, which are infinitely differentiable on $\mathbb{R}$ and have a compact support. A distribution $h \in \mathcal{D}^{\prime}$ is a functional, to which $\langle h, \phi\rangle \in \mathbb{C}$ is associated with every $\phi \in \mathcal{D}$. When $\tilde{f} \in \mathcal{D}^{\prime}$ corresponds to a function $f$, then we put $\langle\tilde{f}, \phi\rangle=\int_{-\infty}^{\infty} f(t) \phi(t) d t$.

Definition 9. Let $\tilde{f}(t)$ be a regular distribution in $\mathcal{D}^{\prime}$ and $f(t)$ be the function corresponding to it. If $h(t) \in \mathcal{D}^{\prime}$ is expressed by $h(t)=D^{n} \tilde{f}(t)$ for $n \in \mathbb{Z}_{>-1}$, then

$$
\langle h, \phi\rangle=\left\langle D^{n} \tilde{f}, \phi\right\rangle=\int_{-\infty}^{\infty} f(t)\left[D_{W}^{n} \phi(t)\right] d t,
$$

for every $\phi(t) \in \mathcal{D}$, where $D_{W}^{n} \phi(t)=(-1)^{n} \frac{d^{n}}{d t^{n}} \phi(t)$.

Lemma 11. $D^{n}$ for $n \in \mathbb{Z}_{>-1}$ are operators in the space $\mathcal{D}^{\prime}$.

In the present study, we consider functions $g_{v}(t)$ and ${ }_{0} D_{R}^{-\lambda} g_{v}(t)$ for $v \in \mathbb{R}_{>0}$ and $\lambda \in \mathbb{R}_{>0}$, and the distributions $\tilde{g}_{v}(t)$ and $D^{-\lambda} \tilde{g}_{v}(t)$ corresponding to them. We then desire to introduce the operator $D_{W}^{-\lambda}$ such that $\left\langle D^{-\lambda} h, \phi\right\rangle=\left\langle h, D_{W}^{-\lambda} \phi\right\rangle$ for $h \in \mathcal{D}^{\prime}$ and $\phi \in \mathcal{D}$. However, we find that $D_{W}^{-\lambda} \phi$ does not belong to $\mathcal{D}$.

In this situation, we consider the problem in the space $\mathcal{D}_{R}^{\prime}$ [12,13]. A regular distribution in $\mathcal{D}_{R}^{\prime}$ is such a distribution that it corresponds to a function which is locally integrable on $\mathbb{R}$ and has a support bounded on the left. The space $\mathcal{D}_{R}$, that is dual to $\mathcal{D}_{R}^{\prime}$, is the space of testing functions, which are infinitely differentiable on $\mathbb{R}$ and have a support bounded on the right.

Definition 10. Definition 9 with $\mathcal{D}^{\prime}$ and $\mathcal{D}$ replaced by $\mathcal{D}_{R}^{\prime}$ and $\mathcal{D}_{R}$, respectively, is valid.

Lemma 12. $D^{v}$ for $v \in \mathbb{C}$ are operators in the space $\mathcal{D}_{R}^{\prime}$.

3.4. Distributions in the Space $\mathcal{D}_{r, R}^{\prime}$

The space $\mathcal{D}_{r, R}$ for $r \in \mathbb{R}$ is such a subspace of $\mathcal{D}_{R}$, that if $\phi(t) \in \mathcal{D}_{r, R}, \phi(t)=0$ for $t \geq r$. A regular distribution $\tilde{f}(t) \in \mathcal{D}_{r, R}^{\prime}$ is such a distribution in $\mathcal{D}_{r, R}^{\prime}$ that it corresponds to a function which is locally integrable on $(-\infty, r)$, and has a support bounded on the left.

Definition 11. Definition 9 with $\mathcal{D}^{\prime}$ and $\mathcal{D}$ replaced by $\mathcal{D}_{r, R}^{\prime}$ and $\mathcal{D}_{r, R}$, respectively, is valid.

Lemma 13. $D^{v}$ for $v \in \mathbb{C}$ are operators in the space $\mathcal{D}_{r, R}^{\prime}$.

Now the rhs of Equation (48) may be expressed as $\int_{-\infty}^{r} f(t)\left[D_{W}^{n} \phi(t)\right] d t$.

\section{Solution of Modified Kummer's DE}

We now study the modified Kummer's DE given by Equation (5). We define $p_{K}(t, s)$ by

$$
p_{K}(t, s)=t \cdot s^{2}+(c-b t) s-a b=t\left(s^{2}-b s\right)+c s-a b .
$$


Then Equation (5) is expressed as $p_{K}\left(t, \frac{d}{d t}\right) u(t)=0, t>0$, and the DE which corresponds to Equation (30) is

$$
p_{K}\left(-\frac{d}{d s}, s\right) \hat{u}(s)=-\frac{d}{d s}\left[\left(s^{2}-b s\right) \hat{u}(s)\right]+(c s-a b) \hat{u}(s)=-(1-c) u_{0},
$$

where the rhs is evaluated by using the first equality and Equations (24) and (26) as

$$
\left\langle p_{K}\left(-\frac{d}{d s}, s\right) \hat{u}(s)\right\rangle_{z}=-\frac{d}{d s}\left(u_{1}+u_{0} \cdot s-b \cdot u_{0}\right)+c \cdot u_{0}=-u_{0}+c \cdot u_{0} .
$$

4.1. Solution Satisfying $(1-c) u_{0}=0$

When $(1-c) u_{0}=0$, the rhs of Equation (50) is 0 , and the solution of Equation (50) is given by

$$
\begin{aligned}
\hat{u}(s) & =C s^{a-1}(s-b)^{-1-a+c}=C s^{-2+c} \sum_{k=0}^{\infty} \frac{(1+a-c)_{k}}{k !}\left(\frac{b}{s}\right)^{k} \\
& =C s^{-2+c} \cdot{ }_{1} F_{0}\left(1+a-c ; ; \frac{b}{s}\right), \quad|s|>|b|,
\end{aligned}
$$

where ${ }_{1} F_{0}(a ; z)=\sum_{k=0}^{\infty} \frac{(a)_{k}}{k !} z^{k}$ and $C$ is an arbitrary constant. If $1-c \notin \mathbb{Z}_{<1}, \hat{u}(s)$ is of the form of Equation (9), and we can take its inverse Laplace transform. Then, choosing $C=\Gamma(2-c)$, we obtain

$$
u(t)=\Gamma(2-c) t^{1-c} \sum_{k=0}^{\infty} \frac{(1+a-c)_{k}}{k ! \Gamma(2-c+k)} b^{k} t^{k}=t^{1-c} \cdot{ }_{1} F_{1}(1+a-c ; 2-c ; b t), \quad t>0,
$$

by using the formula $\Gamma(2-c+k)=\Gamma(2-c)(2-c)_{k}$. This is the solution given by Equation (7).

By Corollary 4 , we confirm the following lemma.

Lemma 14. If $1-c \notin \mathbb{Z}_{<0}$, then $u(t)$ given by Equation (53) is a solution of Equation (5).

The derivation of this lemma based on the distribution theory is as follows. When $1-c \notin \mathbb{Z}_{<0}$, $\tilde{u}(t)=\hat{u}(D) \delta(t)$ takes the form of Equation (43), and satisfies $p_{K}\left(-\frac{\partial}{\partial D}, D\right) \hat{u}(D) \delta(t)=(1-c) u_{0}$. $\delta(t)=0$. We put $C=\Gamma(2-c)$, and then the corresponding solution $u(t)$ is given by Equation (53), by Corollary 8.

\subsection{Solution Satisfying $u_{0}=1$}

In [4-6], the other solution given by Equation (6) of Equation (5) is obtained by solving the inhomogeneous Equation (50) for $b \neq 0,1-c \neq 0$ and $u_{0}=1$. We note here that the method using the following lemma with the aid of the solution given by Equation (53), which is already obtained, is easier.

Lemma 15. If $u(a, c ; b t)$ is a solution of Equation (5), $t^{1-c} u(1+a-c, 2-c ; b t)$ is also a solution of the same equation.

Proof We put $u(t)=t^{\lambda} w(t)$ in Equation (5), and then we obtain Equation (5) with $a, c$ and $u$ replaced by $1+a-c, 2-c$ and $w$, respectively, when $\lambda=1-c$. 


\subsection{Solution by the Basic Method}

We note that the solutions of Equations (5) and (50) are obtained by using the basic method of solution, which is explained in [14] (Section 10.3). In that method, the solution of Equation (5) is assumed to be given by

$$
u(t)=t^{\alpha} \sum_{k=0}^{\infty} p_{k} t^{k}
$$

where $\alpha \in \mathbb{C} \backslash \mathbb{Z}_{<1}, p_{k} \in \mathbb{C}$ and $p_{0} \neq 0$. Then, by Theorem 2 , we have

$$
\hat{u}(s)=s^{-\alpha-1} \sum_{k=0}^{\infty} \hat{p}_{k} s^{-k},
$$

where $\hat{p}_{k}=p_{k} \Gamma(\alpha+k+1)$.

In solving Equation (5), Equation (54) is put in it. In solving Equation (50), Equation (55) is put in it. We then note that the condition of $\hat{p}_{0}=p_{0} \Gamma(\alpha+1)=C \neq 0$ requires (i) $\alpha=1-c$ and $\hat{p}_{0}=p_{0} \Gamma(2-c) \neq 0$, or (ii) $\alpha=0$ and $\hat{p}_{0}=p_{0}=u_{0} \neq 0$. Then $\hat{p}_{k}$ or $p_{k}$ for $k \in \mathbb{Z}_{>0}$ are determined by

$$
\begin{aligned}
& \hat{p}_{k}=\hat{p}_{k-1} \frac{\alpha+a+k-1}{\alpha+c+k-1} b=p_{0} \Gamma(\alpha+1) \frac{(\alpha+a)_{k}}{(\alpha+c)_{k}} b^{k} \\
& p_{k}=p_{k-1} \frac{\alpha+a+k-1}{(\alpha+c+k-1)(\alpha+k)} b=p_{0} \frac{(\alpha+a)_{k}}{(\alpha+c)_{k}(\alpha+1)_{k}} b^{k}
\end{aligned}
$$

When $\alpha=0$, we have

$$
\hat{p}_{k}=p_{0} \frac{(a)_{k}}{(c)_{k}} b^{k}, \quad p_{k}=p_{0} \frac{(a)_{k}}{k !(c)_{k}} b^{k}
$$

When $\alpha=1-c$, we have

$$
\hat{p}_{k}=p_{0} \Gamma(2-c) \frac{(1+a-c)_{k}}{k !} b^{k}, \quad p_{k}=p_{0} \frac{(1+a-c)_{k}}{k !(2-c)_{k}} b^{k} .
$$

Using Equation (58) with $\alpha=1-c$ and $p_{0}=1$ in Equations (55) and (54), we obtain Equations (52) and (53), respectively. Using Equation (57) with $\alpha=0$ and $p_{0}=1$ in Equation (54), we obtain Equation (6).

\subsection{Solution by the Modified Nishimoto's Method}

In [25], the solution of Kummer's DE is given by modified Nishimoto's method, where the solution is expressed by $\mathrm{fD}$ of a function. In that paper, the solution given by Equation (53) of Equation (5) is obtained by using Lemma 11 and Proof of Lemma 10 in [25] (Section 3.2).

Remark 6. In Lemmas 9 and 10 in [25], four of Kummer's eight solutions of Kummer's DE are given. We note here that these lemmas are applicable to the modified Kummer's DE, if we introduce the following replacements in Sections 3.1 and 3.2 in [25]. We replace (i): (11) in Lemma 9 and in Proof of Lemma 9 by (18), (ii): $e^{z}$ in Equations (14), (16) and (17) by $e^{\delta \cdot z}$; (iii): 1 and -1 in the column for $\delta_{l}$ in Table 2 by $\delta$ and $-\delta$, respectively; (iv): ${ }_{1} F_{1}(* ; * ; z)$ and ${ }_{1} F_{1}(* ; * ;-z)$ in (16) and (17) by ${ }_{1} F_{1}(* ; * ; \delta \cdot z)$ and ${ }_{1} F_{1}(* ; * ;-\delta \cdot z)$, respectively, and $(\mathrm{v}): \delta_{4}=-\delta_{3}=-1$ in Proof of Lemma 9 by $\delta_{4}=-\delta_{3}=-\delta$. 


\section{Solution of the Hypergeometric DE}

In solving the hypergeometric DE given by Equation (10), we introduce $p_{H}(t, s)$, by

$$
p_{H}(t, s)=t(1-t) \cdot s^{2}+[c-(a+b+1) t] \cdot s-a b=-t^{2} \cdot s^{2}+t\left[s^{2}-(a+b+1) s\right]+c s-a b .
$$

Now, we put $\hat{u}(s)=\mathcal{L}_{S}[u(t)]$ and then the DE which corresponds to Equation (30) is

$$
p_{H}\left(-\frac{d}{d s}, s\right) \hat{u}(s)=-\frac{d^{2}}{d s^{2}}\left[s^{2} \hat{u}(s)\right]-\frac{d}{d s}\left[\left(s^{2}-(a+b+1) s\right) \hat{u}(s)\right]+(c s-a b) \cdot \hat{u}(s)=-(1-c) u_{0},
$$

where the rhs is evaluated by using the first equality and Equations (24) and (26) as

$$
\left\langle p_{H}\left(-\frac{d}{d s}, s\right) \hat{u}(s)\right\rangle_{z}=-u_{0}+c \cdot u_{0} .
$$

Lemma 16. Let $\hat{u}(s)$ be a solution of Equation (60), $\hat{u}(s)=s^{a-1} \hat{w}(s)$ and

$$
p_{W}(t, s)=-t^{2} \cdot s+t(s-(1-a+b))-(a-c) .
$$

Then, $\hat{w}(s)$ is a solution of the following DE:

$$
p_{W}\left(-\frac{d}{d s}, s\right) \hat{w}(s)=-\frac{d^{2}}{d s^{2}}[s \cdot \hat{w}(s)]-\frac{d}{d s}[(s-(1-a+b)) \hat{w}(s)]-(a-c) \hat{w}(s)=-(1-c) u_{0} s^{-a} .
$$

Proof We put $\hat{u}(s)=s^{\lambda} \hat{v}(s)$ in Equation (60). By using $\frac{d}{d s}\left[s^{\lambda} \hat{v}(s)\right]=s^{\lambda}\left[\lambda s^{-1} \hat{v}(s)+\frac{d}{d s} \hat{v}(s)\right]$ and

$$
\frac{d^{2}}{d s^{2}}\left[s^{\lambda} \hat{v}(s)\right]=s^{\lambda}\left\{\lambda(\lambda-1) s^{-2} \hat{v}(s)+2 \lambda \frac{d}{d s}\left[s^{-1} \hat{v}(s)\right]+\frac{d^{2}}{d s^{2}} \hat{v}(s)\right\},
$$

we obtain

$$
\begin{array}{r}
-\frac{d^{2}}{d s^{2}}\left[s^{2} \hat{v}(s)\right]-\frac{d}{d s}[(s-(1-2 \lambda+a+b)) s \cdot \hat{v}(s)]-[(\lambda-c) s+(\lambda-a)(\lambda-b)] \hat{v}(s) \\
=-(1-c) u_{0} s^{-\lambda}
\end{array}
$$

When $\lambda=a$ and $\hat{w}(s)=s \cdot \hat{v}(s)$, this gives Equation (63).

\subsection{Solution Satisfying $(1-c) u_{0}=0$}

In this section, we use the distribution theory in the space $\mathcal{D}_{r, R}^{\prime}$ for $r=1$, so that $\delta_{1}(t)$ appears.

Lemma 17. Let $a-c \notin \mathbb{Z}_{<0}$. Then, we have the following complementary solution (C-solution) of Equation (63):

$$
\hat{w}(s)=K s^{-1-a+c} \sum_{k=0}^{\infty} \frac{(1+b-c)_{k}(1+a-c)_{k}}{k !} s^{-k}=K s^{-1-a+c} \cdot{ }_{2} F_{0}\left(1+b-c, 1+a-c ; ; s^{-1}\right),
$$

where $K$ is any constant, and ${ }_{2} F_{0}(a, b ; ; z)=\sum_{k=0}^{\infty} \frac{(a)_{k}(b)_{k}}{k !} z^{k}$.

Proof For $p_{W}(t, s)$ given by Equation (62), we choose the DE for $w(t)$ as follows:

$$
p_{W}\left(t, \frac{d}{d t}\right) w(t)=\left(t-t^{2}\right) \frac{d}{d t} w(t)-[(1-a+b) t+(a-c)] w(t)=0, \quad t>0 .
$$


The solution of this DE is

$$
\begin{aligned}
w(t) & =C_{1} \cdot t^{a-c}(1-t)^{-1-b+c}=C_{1} \cdot t^{a-c} \sum_{k=0}^{\infty} \frac{(1+b-c)_{k}}{k !} t^{k} \\
& =C_{1} \cdot t^{a-c}{ }_{1} F_{0}(1+b-c ; ; t), \quad 0<t<1,
\end{aligned}
$$

where $C_{1}$ is an arbitrary constant. $\hat{w}(s)$ given by Equation (65) is the term-by-term Laplace transform of this $w(t)$. Theorem 2 and Proposition 5 show that Equation (63) for $(1-c) u_{0}=0$ is satisfied by this $\hat{w}(s)$, since the first equality of Equation (63) shows $\left\langle p_{W}\left(-\frac{d}{d s}, s\right) \hat{w}(s)\right\rangle_{z}=0$,

If $a-c \notin \mathbb{Z}_{<0}$, this $w(t)$ takes the form of Equation (3), and the corresponding $\tilde{w}(t)$, expressed as Equation (43), is given by $\tilde{w}(t)=\hat{w}(D) \delta_{1}(t)$, when $\hat{w}(s)$ is given by Equation (65) and $K=C_{1}$. $\Gamma(1+a-c)$.

Remark 7. We note that $\hat{w}(s)$ given by Equation (65) converges for no value of $s$, but $\tilde{w}(t)=$ $\hat{w}(D) \delta_{1}(t)$ obtained by using this $\hat{w}(s)$ corresponds to Equation (67). Moreover, we note that Equation (67) is obtained from Equation (65) by term-by-term inverse Laplace transform.

We choose $K=\Gamma(2-c)$. Then, by Lemmas 16 and 17,

$$
\hat{u}(s)=s^{a-1} \hat{w}(s)=\Gamma(2-c) s^{-2+c} \cdot{ }_{2} F_{0}\left(1+a-c, 1+b-c ; ; s^{-1}\right)
$$

is a solution of Equation (63). Now, by its term-by-term inverse Laplace transform, or from $\tilde{u}(t)=$ $\hat{u}(D) \delta_{1}(t)=D^{a-1} \hat{w}(D) \delta_{1}(t)$, we obtain $u(t)$ given by Equation (12), if $2-c \notin \mathbb{Z}_{<1}$. We note that $(1-c) u_{0}=0$ for this solution $u(t)$ if $1-c \notin \mathbb{Z}_{<1}$.

Remark 8. Since $\hat{u}(s)=s^{a-1} \hat{w}(s), \tilde{u}(t)=\hat{u}(D) \delta_{1}(t)=D^{a-1} \hat{w}(D) \delta_{1}(t)$, and hence the solution given by Equation (12) is obtained by using Equation (67) in $u(t)={ }_{0} D_{R}^{a-1} w(t)$.

Remark 9. Remark 7 is valid even when $w$, Equations (65) and (67) are replaced by $u$, Equations (68) and (12), respectively.

\subsection{Solution Satisfying $u_{0}=1$}

The other solution given by Equation (11) is obtained by solving the inhomogeneous Equation (63) for $1-c \neq 0$ and $u_{0}=1$. We note here that the method using the following lemma with the solution given by Equation (12), which is already obtained, is easier.

Lemma 18. If $u(a, b, c ; t)$ is a solution of Equation (10), $t^{1-c} \cdot u(1+a-c, 1+b-c, 2-c ; t)$ is also a solution of the same equation.

Proof We put $u(t)=t^{\lambda} w(t)$ in Equation (10), and then we obtain Equation (10) with $a, b, c$ and $u$ replaced by $1+a-c, 1+b-c, 2-c$ and $w$, respectively, when $\lambda=1-c$. 


\subsection{Solution by the Basic Method}

The statements in Section 4.3 are valid even if we replace Equations (50), (5), (52) and (53) by Equations (60), (10), (68) and (12), respectively. Here, Equations (56) (58) also must be replaced by

$$
\begin{aligned}
& \hat{p}_{k}=\hat{p}_{k-1} \frac{(\alpha+a+k-1)(\alpha+b+k-1)}{\alpha+c+k-1}=p_{0} \Gamma(\alpha+1) \frac{(\alpha+a)_{k}(\alpha+b)_{k}}{(\alpha+c)_{k}}, \\
& p_{k}=p_{k-1} \frac{(\alpha+a+k-1)(\alpha+b+k-1)}{(\alpha+c+k-1)(\alpha+k)}=p_{0} \frac{(\alpha+a)_{k}(\alpha+b)_{k}}{(\alpha+c)_{k}(\alpha+1)_{k}}
\end{aligned}
$$

When $\alpha=0$, we have

$$
\hat{p}_{k}=p_{0} \frac{(a)_{k}(b)_{k}}{(c)_{k}}, \quad p_{k}=p_{0} \frac{(a)_{k}(b)_{k}}{k !(c)_{k}}
$$

When $\alpha=1-c$, we have

$$
\hat{p}_{k}=p_{0} \Gamma(2-c) \frac{(1+a-c)_{k}(1+b-c)_{k}}{k !}, \quad p_{k}=p_{0} \frac{(1+a-c)_{k}(1+b-c)_{k}}{k !(2-c)_{k}}
$$

Using Equation (71) with $\alpha=1-c$ and $p_{0}=1$ in Equations (54) and (55), we obtain Equations (12) and (68), respectively. Using Equation (70) with $\alpha=0$ and $p_{0}=1$ in Equation (54), we obtain Equation (11).

\subsection{Solution by the Modified Nishimoto's Method}

In [26], the solution of the hypergeometric DE is given by modified Nishimoto's method, where the solution is expressed by $\mathrm{fD}$ of a function. In that paper, Kummer's 24 solutions of the DE are obtained by that method.

\section{Solution of Bessel's DE}

We now take up Bessel's DE:

$$
t^{2} \cdot \frac{d^{2}}{d t^{2}} u(t)+t \cdot \frac{d}{d t} u(t)+\left(t^{2}-v^{2}\right) \cdot u(t)=0, \quad t>0
$$

where $v \in \mathbb{C}$ is a constant. The Bessel functions $J_{ \pm v}(t)$ are solutions of this DE, where

$$
J_{v}(t)=\left(\frac{t}{2}\right)^{v} \sum_{k=0}^{\infty} \frac{(-1)^{k}}{k ! \Gamma(v+k+1)}\left(\frac{t^{2}}{4}\right)^{k}=\left(\frac{t}{2}\right)^{v} \frac{1}{\Gamma(v+1)}{ }_{0} F_{1}\left(; v+1 ;-\frac{t^{2}}{4}\right),
$$

for $v \notin \mathbb{Z}_{<0}$, where ${ }_{0} F_{1}(; c ; z)=\sum_{k=0}^{\infty} \frac{1}{k !(c)_{k}} z^{k}$.

In order to obtain the solutions given by Equation (73) by the present method, we put $x=t^{2}$ and $u(t)=t^{\lambda} v(x)$ in Equation (72). Then, if $\lambda=v$, we obtain the DE for $v(x)$ :

$$
p_{B}\left(x, \frac{d}{d x}\right) v(x)=4 x \cdot \frac{d^{2}}{d x^{2}} v(x)+4(v+1) \cdot \frac{d}{d x} v(x)+v(x)=0, x>0,
$$

and if $\lambda=-v$, we obtain the same equation with $v$ replaced by $-v$.

When $\lambda=v$, by Theorem 2, the DE satisfied by the AC-Laplace transform $\hat{v}(s)$ of $v(x)$ is given by

$$
p_{B}\left(-\frac{d}{d s}, s\right) \hat{v}(s)=-4 \frac{d}{d s}\left[s^{2} \hat{v}(s)\right]+[4(v+1) s+1] \hat{v}(s)=4 v v_{0} .
$$


The solution satisfying $v v_{0}=0$ is given by

$$
\hat{v}(s)=C s^{\nu-1} e^{-1 /(4 s)}=C s^{v-1} \sum_{k=0}^{\infty} \frac{(-1)^{k}}{k !} \frac{1}{(4 s)^{k}},
$$

where $C$ is an arbitrary constant. By the inverse Laplace transform, we obtain

$$
v(x)=C x^{-v} \sum_{k=0}^{\infty} \frac{(-1)^{k}}{k ! \Gamma(k-v+1)}\left(\frac{x}{4}\right)^{k}=C x^{-v} \frac{1}{\Gamma(1-v)} \cdot{ }_{0} F_{1}\left(; 1-v ;-\frac{x}{4}\right) .
$$

This satisfies $v v_{0}=0$ if $v \notin \mathbb{Z}_{>0}$. By Corollary 4 , this $v(x)$ is a solution of Equation (74) if $v \notin \mathbb{Z}_{>0}$.

Using this in $u(t)=t^{v} v\left(t^{2}\right)$ and putting $C=2^{v}$, we have $u(t)=J_{-v}(t)$. When $\lambda=-v$, we obtain $u(t)=J_{v}(t)$.

\section{Solution of Hermite's DE}

We now take up Hermite's DE:

$$
\frac{d^{2}}{d t^{2}} u(t)-t \cdot \frac{d}{d t} u(t)+n \cdot u(t)=0, \quad t>0,
$$

where $n \in \mathbb{Z}_{>-1}$ is a constant. The Hermite polynomial $H e_{n}(t)$ and the Hermite function of the second kind $h e_{n}(t)$ are solutions of this DE. In [11] (p. 82), they are expressed as

$$
\begin{aligned}
& H e_{2 l}(t)=\frac{(-1)^{l}}{2^{l}} \frac{(2 l) !}{l !} \cdot{ }_{1} F_{1}\left(-l ; \frac{1}{2} ; \frac{t^{2}}{2}\right), \quad H e_{2 l+1}(t)=\frac{(-1)^{l}}{2^{l}} \frac{(2 l+1) !}{l !} \cdot t \cdot{ }_{1} F_{1}\left(-l ; \frac{3}{2} ; \frac{t^{2}}{2}\right), \\
& h e_{2 l}(t)=(-1)^{l} 2^{l} l ! \cdot t \cdot{ }_{1} F_{1}\left(\frac{1}{2}-l ; \frac{3}{2} ; \frac{t^{2}}{2}\right), \quad h e_{2 l+1}(t)=(-1)^{l+1} 2^{l} l ! \cdot{ }_{1} F_{1}\left(-\frac{1}{2}-l ; \frac{1}{2} ; \frac{t^{2}}{2}\right),
\end{aligned}
$$

for $l \in \mathbb{Z}_{>-1}$.

In order to obtain these solutions by the present method, we put $x=t^{2}$ and $u(t)=v(x)$ in Equation (78). Then, we obtain the DE for $v(x)$ :

$$
x \cdot \frac{d^{2}}{d x^{2}} v(x)+\left(\frac{1}{2}-\frac{1}{2} x\right) \cdot \frac{d}{d x} v(x)+\frac{1}{4} n \cdot v(x)=0, \quad x>0 .
$$

This DE is Equation (5) with $t, u(t), c, b$ and $a$ replaced by $x, v(x), \frac{1}{2}, \frac{1}{2}$ and $-\frac{1}{2} n$, respectively. Corresponding to the solutions given by Equations (6) and (7) of Equation (5), we have the following solutions of Equation (81):

$$
v_{n, 1}(x)={ }_{1} F_{1}\left(-\frac{1}{2} n ; \frac{1}{2} ; \frac{x}{2}\right), \quad v_{n, 2}(x)=x^{1 / 2} \cdot{ }_{1} F_{1}\left(\frac{1}{2}-\frac{1}{2} n ; \frac{3}{2} ; \frac{x}{2}\right) .
$$

The solutions given by Equations (79) and (80) of Equation (78) are given by

$$
\begin{aligned}
H e_{2 l}(t) & =C_{1} v_{2 l, 1}\left(t^{2}\right), & H e_{2 l+1}(t)=C_{2} v_{2 l+1,2}\left(t^{2}\right), \\
h e_{2 l}(t) & =C_{3} v_{2 l, 2}\left(t^{2}\right), & h e_{2 l+1}(t)=C_{4} v_{2 l+1,1}\left(t^{2}\right),
\end{aligned}
$$

for $l \in \mathbb{Z}_{>-1}$, where $C_{k}$ for $k \in \mathbb{Z}_{[1,4]}$ are constants.

\section{Conclusions}

In the present paper, we are concerned with the problem of obtaining a solution $u(t)$ of a DE with polynomial coefficients. 
We know that by the basic method of solution [14] (Section 10.4), we usually obtain solutions in the form of Equation (54) which are a power of $t$ multiplied by a power series in $t$. In the present paper, we are interested in obtaining the solutions with the aid of AC of Riemann-Liouville fD along with distribution theory or the Laplace transform or its AC.

We then set up the DE satisfied by the AC-Laplace transform, $\hat{u}(s)$, of $u(t)$. The obtained DE for $\hat{u}(s)$ is found to be a DE with polynomial coefficients.

We now obtain the solution in the form of Equation (55) which is a power of $s$ multiplied by a power series in $s^{-1}$, by solving the DE for $\hat{u}(s)$. When it converges at large $|s|$, it is the Laplace transform of a solution of the DE for $u(t)$ or its AC.

In Section 4, we obtain such a solution $\hat{u}(s)$ that the series in it converges at $|s|>|b|$ for $b \neq 0$. Then, we obtain the solution $u(t)$ by term-by-term inverse Laplace transform by writing the distribution associated with the solution $u(t)$, by using the obtained $\hat{u}(s)$. In Section 6 , another example is given.

In Section 5, we obtain such a solution $\hat{u}(s)$ that the series in it converges for no value of $s$. Then, we obtain the solution $u(t)$ by writing the distribution $\tilde{u}(t)=\hat{u}(D) \delta_{1}(t)$ associated with the solution $u(t)$, by using the obtained non-convergent series of $s^{-1}$ for $\hat{u}(s)$. The result is seen to be obtained by term-by-term inverse Laplace transform of $\hat{u}(s)$, as mentioned in Section 3.2.

We may conclude the study in this paper as follows. When we desire to obtain the solution $u(t)$ of a linear DE with polynomial coefficients, in the form of Equation (3), we can obtain it, by setting up the DE for the AC-Laplace transform $\hat{u}(s)$, obtaining its solution in the form of Equation (9), and then taking its term-by-term inverse Laplace transform.

We can obtain the solution of the DE for $\hat{u}(s)$ by the basic method, obtaining it in the form of Equation (55). Comparing the solutions of the DE for $u(t)$ and $\hat{u}(s)$, obtained by the basic method of solution, we find that the solution $\hat{u}(s)$ is the term-by-term Laplace transform of $u(t)$. Thus, we obtain $u(t)$ by the term-by-term inverse Laplace transform of $\hat{u}(s)$, when the latter is obtained.

Acknowledgments: Section 1.2 and the paragraphs related with the basic references [21-24] on distribution theory in Section 3.3 are written in response to the recommendations of the reviewers of this paper.

Author Contributions: Stimulated by Yosida's work [1,2], a number of papers [4-6] were written on the solution of Laplace's DE. But the research there are mainly focussed on Kummer's DE. In this situation, Tohru Morita came to the idea that distributions in the space $\mathcal{D}_{r, R}^{\prime}$ must be useful in solving the hypergeometric DE. Based on the idea, Tohru Morita and Ken-ichi Sato worked together to write this paper.

Conflicts of Interest: The authors declare no conflict of interest.

\section{References}

1. Yosida, K. The Algebraic Derivative and Laplace's Differential Equation. Proc. Jpn. Acad. Ser. A 1983, 59, $1-4$.

2. Yosida, K. Operational Calculus; Springer-Verlag: New York, NY, USA, 1982.

3. Mikusiński, J. Operational Calculus; Pergamon Press: London, UK, 1959.

4. Morita, T.; Sato, K. Remarks on the Solution of Laplace's Differential Equation and Fractional Differential Equation of That Type. Appl. Math. 2013, 4, 13-21.

5. Morita, T.; Sato, K. Solution of Laplace's Differential Equation and Fractional Differential Equation of That Type. Appl. Math. 2013, 4, 26-36.

6. Morita, T.; Sato, K. Solution of Differential Equations with the Aid of Analytic Continuation of Laplace Transform. Appl. Math. 2014, 5, 1309-1319.

7. Lavoie, J.L.; Tremblay, R.; Osler, T.J. Fundamental Properties of Fractional Derivatives Via Pochhammer Integrals. In Fractional Calculus and Its Applications, Ross, B., Ed.; Lecture Notes in Mathematics; Springer: Berlin, Germany; Heidelberg, Germany, 1975; Volume 457, pp. 327-356.

8. Lavoie, J.L.; Osler, T.J.; Tremblay, R. Fractional Derivatives and Special Functions. SIAM Rev. 1976, 18, 240-268.

9. Morita, T.; Sato, K. Liouville and Riemann-Liouville Fractional Derivatives via Contour Integrals. Fract. Calc. Appl. Anal. 2013, 16, 630-653. 
10. Abramowitz, M.; Stegun, I.A. Handbook of Mathematical Functions with Formulas, Graphs and Mathematical Tables; Dover Publ., Inc.: New York, NY, USA, 1972.

11. Magnus, M.; Oberhettinger, F. Formulas and Theorems for the Functions of Mathematical Physics; Chelsea Publ. Co.: New York, NY, USA, 1949.

12. Morita, T.; Sato, K. Solution of Fractional Differential Equation in Terms of Distribution Theory. Interdiscip. Inf. Sci. 2006, 12, 71-83.

13. Morita, T.; Sato, K. Neumann-Series Solution of Fractional Differential Equation. Interdiscip. Inf. Sci. 2010, $16,127-137$.

14. Whittaker, E.T.; Watson, G.N. A Course of Modern Analysis; Cambridge U.P.: Cambridge, UK, 1935.

15. He, J.H. A Tutorial Review on Fractal Spacetime and Fractional Calculus. Int. J. Theor. Phys. 2014, 53, 3698-3718.

16. Liu, F.J.; Li, Z.B.; Zhang, S.; Liu, H.Y. He's Fractional Derivative for Heat Conduction in a Fractal Medium Arising in Silkworm Cocoon Hierarchy. Therm. Sci. 2015, 19, 1155-1159.

17. Kumar, D.; Singh, J.; Kumar, S. A Fractional Model of Navier-Stokes Equation Arising in Unsteady Flow of a Viscous Fluid. J. Assoc. Arab Univ. Basic Appl. Sci. 2015, 17, 14-17.

18. Kumar, D.; Singh, J.; Kumar, S.; Sushila; Singh, B.P. Numerical Computation of Nonlinear Shock Wave Equation of Factional Order. Ain Shams Eng. J. 2015, 6, 605-611.

19. He, J.H.; Elagan, S.K.; Li, Z.B. Geometrical Explanation of the Fractional Complex Transform and Derivative Chain Rule for Fractional Calculus. Phys. Lett. A 2013, 376, 257-259.

20. Jia, S.M.; Hu, M.S.; Chen, Q.L.; Jia, Z.J. Exact Solution of Fractional Nizhnik-Novikov-Veselov Equation. Therm. Sci. 2014, 18, 1715-1717.

21. Schwartz, L. Théorie des Distributions; Hermann: Paris, France, 1966.

22. Gelfand, I.M.; Silov, G.E. Generalized Functions; Academic Press Inc.: New York, NY, USA, 1964; Volume 1.

23. Vladimirov, V.S. Methods of the Theory of Generalized Functions; Taylor \& Francis Inc.: New York, NY, USA, 2002.

24. Zemanian, A.H. Distribution Theory and Transform Analysis; Dover Publ., Inc.: New York, NY, USA, 1965.

25. Morita, T.; Sato, K. Asymptotic Expansions of Fractional Derivatives and Their Applications. Mathematics 2015, 3, 171-189.

26. Morita, T.; Sato, K. Kummer's 24 Solutions of the Hypergeometric Differential Equation with the Aid of Fractional Calculus. Adv. Pure Math. 2016, 6, 180-191.

(C) 2016 by the authors; licensee MDPI, Basel, Switzerland. This article is an open access article distributed under the terms and conditions of the Creative Commons by Attribution (CC-BY) license (http:/ / creativecommons.org/licenses/by/4.0/). 